\title{
COEFICIENTE DE TRANSFERÊNCIA DE CALOR NA INTERFACE METAL/MOLDE E VARIÁVEIS TÉRMICAS NA SOLIDIFICAÇÃO DIRECIONAL HORIZONTAL DA LIGA AI-6\%Cu
}

\author{
O. L. Rocha ${ }^{1}$, J. M. Dias Filho ${ }^{1}$, T. A. Costa ${ }^{2}$, A. J. Vasconcelos ${ }^{1}$, A. L. Moreira ${ }^{3}$ e R. Kikushi ${ }^{1}$ \\ ${ }^{1}$ Instituto Federal de Educação, Ciência e Tecnologia do Pará \\ ${ }^{2,3}$ Universidade Federal do Pará \\ E-mail: alsm@ufpa.br ${ }^{3}$
}

Artigo submetido em junho/2013 e aceito em novembro/2013

\section{RESUMO}

O objetivo deste trabalho é determinar o coeficiente de transferência de calor na interface metal/molde $\left(h_{i}\right)$ da liga Al-6\%Si solidificada direcionalmente em um sistema horizontal refrigerado a água bem como as varáveis térmicas de solidificação velocidade da isoterma liquidus $\left(V_{L}\right)$ e taxa de resfriamento $\left(T_{R}\right)$. Um dispositivo experimental de solidificação horizontal foi desenvolvido e amostras da liga foram obtidas sob condições transientes de fluxo de calor. As variáveis térmicas são calculadas a partir dos perfis de temperatura obtidos para cinco termopares inseridos no metal que permitem correlacionar a posição da isoterma liquidus $(P)$ com o tempo de solidificação. Um método baseado no confronto entre os perfis de temperatura teóricos e experimentais foi utilizado para determinar os valores $h_{\mathrm{i}}$. Para o cálculo dos perfis teóricos, aplicou-se um modelo numérico de transferência de calor e massa, consagrado na literatura, para solidificação direcional. Leis experimentais em forma de potência do tipo $h_{i}=$ $A_{1}(t)^{-n 1}, V_{L}=A_{2}(P)^{-n 2}$ e $T_{R}=A_{3}(P)^{-n 3}$ foram obtidas. Um estudo comparativo entre os valores de $h_{i}$ obtidos neste trabalho e aqueles propostos na literatura, durante a solidificação unidirecional vertical ascendente das ligas investigadas, também é apresentado.

PALAVRAS-CHAVE: Solidificação Direcional Horizontal, Coeficiente de Extração de Calor, Variáveis Térmicas, Regime Transitório de Extração de Calor

\section{HEAT TRANSFER COEFFICIENT AT METAL/MOLD INTERFACE ABSTRACT AND THERMAL VARIABLES DURING HORIZONTAL SOLIDIFICATION OF Al-6WT.\%Cu Alloy}

\begin{abstract}
The aim of this study is to determine the metal-mold heat transfer coefficient $\left(h_{i}\right)$ of Al-6\%wt.Cu alloy as well as theoretical values of solidification thermal variables such as tip growth rates $\left(V_{L}\right)$ and cooling rates $\left(T_{R}\right)$. A water-cooled solidification experimental apparatus has been developed, and specimens have been solidified under unsteady state heat flow conditions. The thermal variables were calculated from the temperature profiles obtained for five thermocouples located within the metal which allowed to correlate the position of liquidus isotherm from the metal-mold interface $(P)$ with the
\end{abstract}

solidification time. A method based on the comparison between the theoretical and experimental temperature profiles was used to determine the $h_{i}$ values. A numerical model of heat and mass transfer which is applied to directional solidification was used to calculate the theoretical profiles. Experimental laws expressed as a power function of time given by $h_{i}=A_{1}(t)^{-n 1}, V_{L}=A_{2}(P)^{-n 2}$ and $T_{R}=A_{3}(R)^{-n 3}$ were obtained. $A$ comparison between the values of $h_{i}$ obtained in this work and those proposed in the literature during the upward vertical unidirectional solidification of the alloy investigated is also presented.

KEYWORDS: Horizontal Directional Solidification, Heat Transfer Coefficient, Thermal Variables, UnsteadyState Conditions 


\section{COEFICIENTE DE TRANSFERÊNCIA DE CALOR NA INTERFACE METAL/MOLDE E VARIÁVEIS TÉRMICAS NA SOLIDIFICAÇÃO DIRECIONAL HORIZONTAL DA LIGA AI-6\%Cu}

\section{INTRODUÇÃO}

A solidificação pode ser definida como o fenômeno de transformação da fase líquida para a fase sólida, sendo acompanhada pela liberação de calor latente, que decorre da troca de energia térmica através dos métodos de transferência de calor conhecidos, ou seja, condução, convecção e radiação bem como da transferência newtoniana. Sendo assim, é essencial a análise desses modos de transferência de calor durante o processo de solidificação uma vez que através desta é possível determinar a distribuição de temperaturas no sistema metal/molde e a cinética de solidificação (Garcia, 2007). A Figura 1 apresenta um esquema ilustrativo de um elemento de referência do sistema metal/molde durante a solidificação de um lingote, em que são mostrados todos os modos de transferência de calor que podem ocorrer ao longo da solidificação unidirecional, quais sejam: condução térmica no metal e no molde; transferência newtoniana na interface metal/molde; conveç̧ão no metal líquido e na interface molde/ambiente e radiação térmica do molde para o meio ambiente.

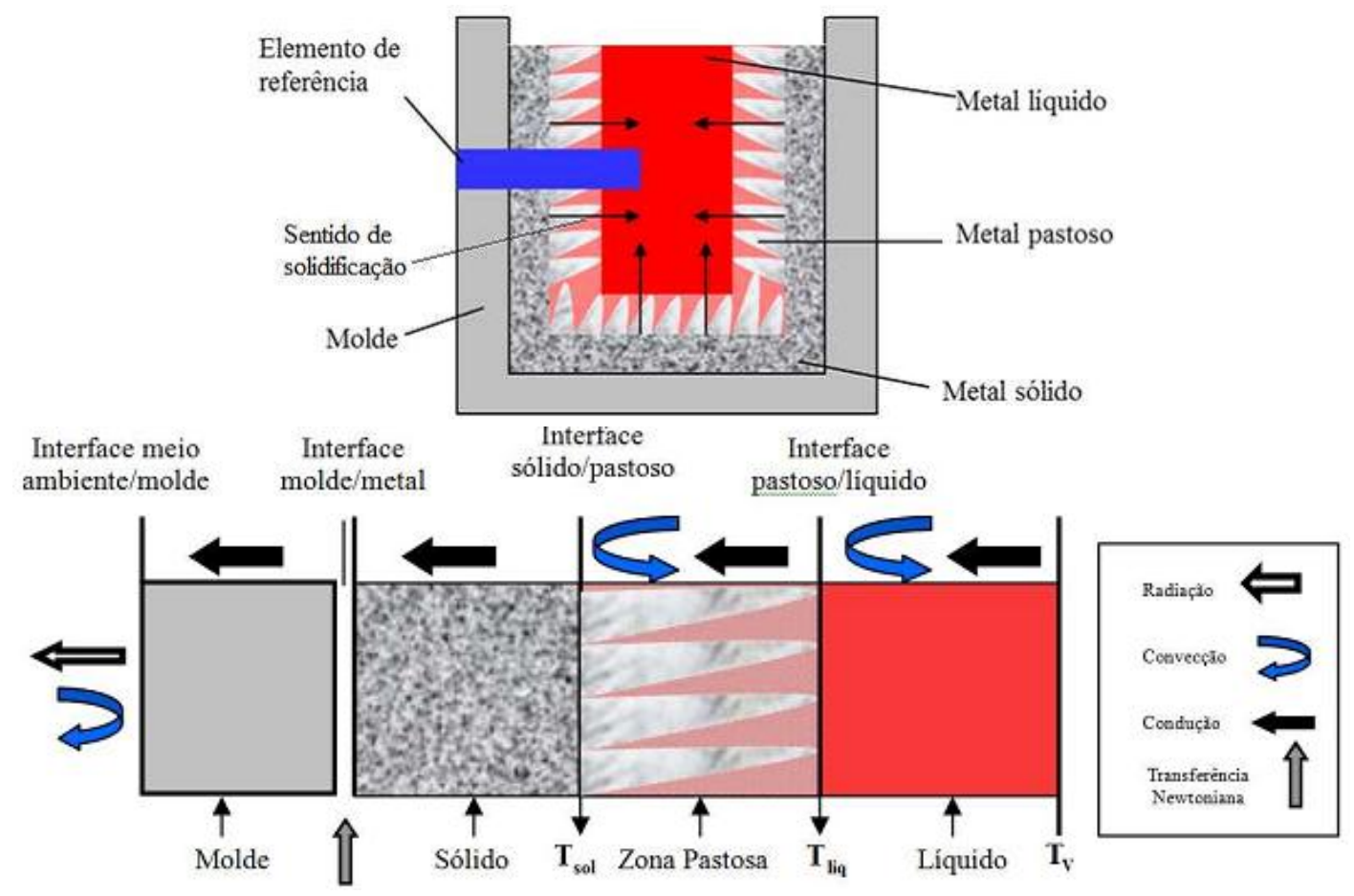

Figura 1 - Elemento de referência representativo do sistema metal/molde. (Meza, 2012).

Na prática, utiliza-se uma temperatura de vazamento $\left(T_{v}\right)$ do metal líquido em moldes acima da temperatura liquidus $\left(T_{L}\right)$ a fim de permitir a acomodação do metal à geometria do molde antes do início da solidificação. O calor sensível proveniente do superaquecimento, que é a diferença entre a temperatura de vazamento e a temperatura liquidus, $\left(\Delta T=T_{V}-T_{L}\right)$, e o calor 
latente liberado na fronteira sólido/liquido são transferidos através do metal solidificado, da interface metal/molde e do molde para o meio ambiente.

Fundamentalmente, as características mecânicas do material dependem das condições assumidas no processo de solidificação. O tamanho, orientação preferencial de crescimento e morfologia dos grãos, os espaçamentos interdendríticos, as heterogeneidades de composição química, o tamanho, forma e distribuição das inclusões e a presença de porosidade entre outros fatores, são decisivos na formação da macroestrutura e da microestrutura do material influenciando, consequentemente, na determinação de suas propriedades. Para a indústria que busca a fabricação em larga escala de produtos com propriedades mecânicas superlativas, o conhecimento da influência que as variáveis térmicas exercem na formação das estruturas solidificadas permite um melhor controle dos processos de fundição uma vez que as mesmas determinam a qualidade dos produtos obtidos, ratificando a relevância científica e tecnológica do fenômeno da solidificação dos materiais metálicos como um importante viés de investigação do campo industrial.

\section{Coeficiente de transferência de calor na interface metal/molde}

Uma vez que o processo de solidificação se inicia com o material no estado líquido, o mesmo necessita de um molde que proporcione a geometria desejada, e que ao mesmo tempo retire o calor liberado pela transformação líquido/sólido (Garcia 2007; Meza, 2012). Este metal ao entrar em contato as paredes do molde, promoverá um fenômeno de resistência de contato, conhecido como resistência newtoniana, que é decorrente de vários fatores dentre os quais podem ser citados a afinidade físico-química entre o material do molde e o material a ser solidificado, a contração do metal e a expansão do molde. Além disso, o contato na fronteira metal/molde não é perfeito, uma vez que a usinagem de acabamento gera uma microgeometria, que propicia o surgimento de rugosidades nas regiões de contato físico entre o metal e o molde e, consequentemente, o molhamento pelo metal líquido na parede interna do molde não é perfeito.

Esses fatores em conjunto, promovem transferência de calor por condução através dos pontos de contato e dos gases aprisionados nos espaços criados, e tanto por convecção como por radiação, entre as duas superfícies separadas.

Portanto, a perda de calor inicial, não é somente regulada pela capacidade térmica de armazenamento do material do molde, mas também pelas condições de transferência de calor do metal e, particularmente, na interface metal/molde. As partículas sólidas encontram-se somente em contato em pontos isolados e a área real de contato é somente uma pequena fração da área nominal, conforme apresentado na Figura 2. 


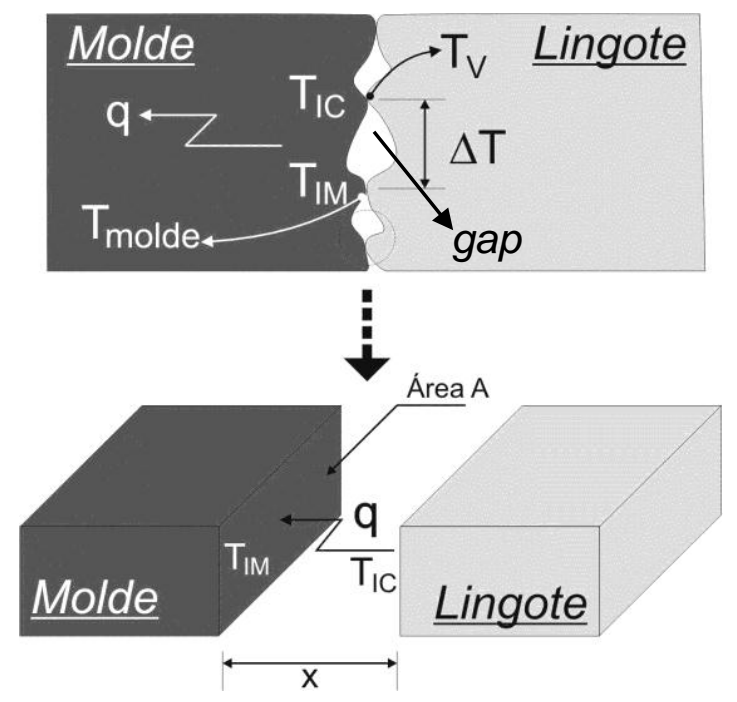

Figura 2- Fluxo de calor através da interface metal/molde. (Spinelli, 2004).

Parte do fluxo de calor segue os caminhos de contato real, todavia, o calor remanescente deve ser transmitido por meios intersticiais gasosos e não-gasosos entre as rugosidades de ambas as superfícies. Os interstícios são limitados no tamanho e desta forma a convecção pode ser desprezada. Se as diferenças de temperaturas não são extremas, a radiação também não é tão significativa e, por conseguinte, a maior parte da energia fluirá apenas por condução através da área real de contato físico.

O fluxo de calor através da interface metal/molde, conforme apresentado esquematicamente na Figura 2, pode ser caracterizado por um coeficiente médio macroscópico de transferência de calor, dado por:

$$
\mathrm{h}_{\mathrm{i}}=\frac{\mathrm{q}}{\mathrm{A} \cdot\left(\mathrm{T}_{\mathrm{IC}}-\mathrm{T}_{\mathrm{IM}}\right)}
$$

onde q é o fluxo de calor global através da interface, $T_{I C}$ e $T_{I M}$ são, respectivamente, as temperaturas superficiais do lingote e do molde e $\mathrm{A}$ é a área.

No entanto, as temperaturas $T_{I C}$ e $T_{I M}$ são difíceis de serem medidas pois a localização exata de termopares na interface não se constitui em uma tarefa trivial. Para superar este obstáculo experimental, os métodos de cálculo de $h_{i}$ existentes na literatura são baseados no conhecimento e aplicação de outras condições.

No caso de moldes refrigerados a água, o fluxo de calor global é afetado por uma série de resistências térmicas, conforme apresentado na Figura 3. 


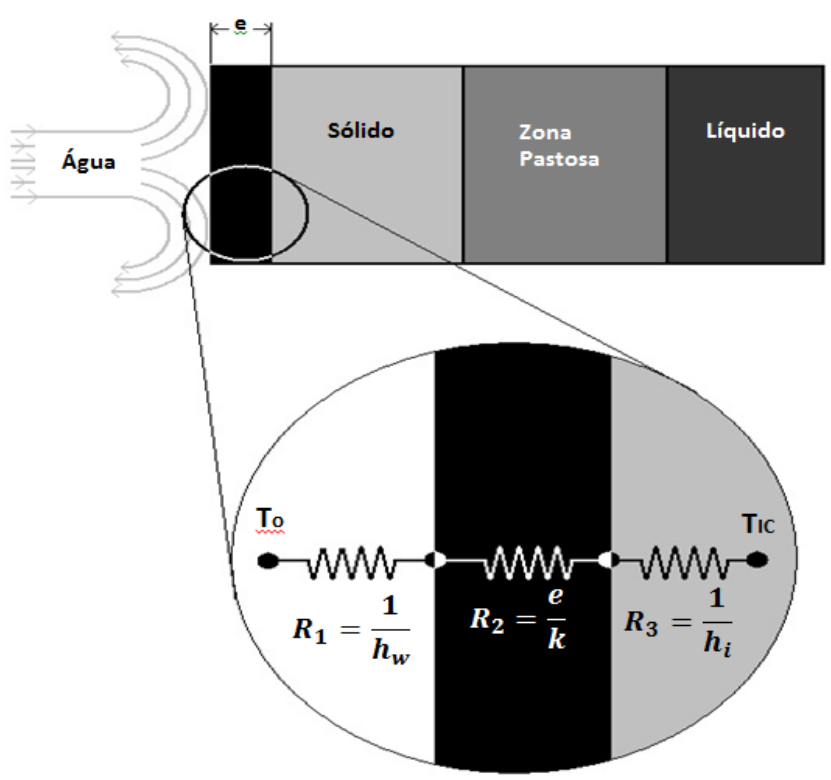

Figura 3 - Resistências térmicas na interface metal/molde em molde refrigerado. (Adaptado de Santos et al., 2001)

A resistência interfacial entre as superfícies do lingote e do molde são geralmente as maiores, e a resistência térmica global $\frac{1}{h_{g}}$ é expressa por:

$\frac{1}{h_{g}}=\frac{1}{h_{W}}+\frac{e}{k}+\frac{1}{h_{i}}$

equação (2)

onde $h_{\mathrm{g}}$ é o coeficiente global de transmissão de calor entre a superfície do lingote e o fluido refrigerante, e é a espessura da base do molde e, finalmente, $\mathrm{h}_{\mathrm{W}}$ é o coeficiente de transmissão de calor molde/fluido de refrigeração. O fluxo de calor médio da superfície do lingote até a água que refrigera o sistema é então dado por:

$q=h_{g} \cdot\left(T_{I C}-T_{0}\right)$,

equação (3)

onde $\mathrm{T}_{0}$ é a temperatura da água.

A disponibilidade de valores de $\mathrm{h}_{\mathrm{g}}$ na literatura é relativamente escassa, e as características de alta diversidade dos sistemas metal/molde implicam na necessidade do desenvolvimento de metodologias para sua determinação. Pode-se mencionar quatro metodologias de determinação de $h_{g}$, que individualmente estão apoiadas em diferentes abordagens do processo básico da solidificação tanto de metais como de ligas metálicas binárias, a saber: cinética de solidificação unidirecional controlada; medidas de parâmetros da microestrutura de solidificação; medida de temperatura e vazão em moldes refrigerados; e confronto de perfis térmicos teóricos/experimentais (Santos et al., 2001; Rocha et al., 2003; Garcia 2007).

O método do confronto teórico/experimental de perfis térmicos, que é uma forma indireta de determinação de $h_{i}$, foi selecionado para ser empregado no presente trabalho. 0 mesmo consiste em mapear experimentalmente as temperaturas em determinados pontos do molde e do metal ao longo da solidificação e, posteriormente, confrontar os perfis de temperatura ou curvas de resfriamento experimentais com as curvas teóricas simuladas através 
de um modelo numérico de solidificação. Um modelo numérico de solidificação, baseado no método de diferenças finitas (MDF) (Garcia 2007), é utilizado para obtenção da curva teórica. 0 respectivo modelo encontra-se detalhado no trabalho desenvolvido por Santos et al., (2001). Recentemente, Ferreira (2004) aprimorou o referido modelo, incorporando na modelagem matemática os efeitos convectivos e, assim, ampliando a utilização do mesmo para outros sistemas e configurações de dispositivos de solidificação, como os sistemas vertical descendente e horizontal.

A Tabela 1 representa resultados experimentais de $h_{i}$, obtidos na literatura para ligas Al$\mathrm{Cu}$, considerando diversos sistemas de solidificação direcional vertical, cujos trabalhos utilizaram o método empregado neste trabalho.

Tabela 1 - Resultados de $h_{i}$ para ligas de Al-Cu solidificadas unidirecionalmente.

\begin{tabular}{|c|c|c|c|}
\hline Sistema de Solidificação & Ligas de Alumínio & $h_{i}=A t^{-a}\left[W / m^{2} K\right]$ & Referência \\
\hline \multirow{10}{*}{ Vertical Ascendente } & $\mathrm{Al}-2 \% \mathrm{Cu}$ & $1850(t)^{-0,14}$ & \multirow{4}{*}{ Siqueira (2002) } \\
\hline & $\mathrm{Al}-5 \% \mathrm{Cu}$ & $2400(t)^{-0,27}$ & \\
\hline & $\mathrm{Al}-8 \% \mathrm{Cu}$ & $5100(t)^{-0,33}$ & \\
\hline & $\mathrm{Al}-10 \% \mathrm{Cu}$ & $5700(t)^{-0,33}$ & \\
\hline & $\mathrm{Al}-5 \% \mathrm{Cu}$ & $5500(t)^{-0,18}$ & \multirow{3}{*}{ Rocha (2003) } \\
\hline & $\mathrm{Al}-8 \% \mathrm{Cu}$ & $6500(t)^{-0,18}$ & \\
\hline & $\mathrm{Al}-15 \% \mathrm{Cu}$ & $9000(t)^{-0,18}$ & \\
\hline & $\mathrm{Al}-4,5 \% \mathrm{Cu}$ & $9500(t)^{-0,07}$ & \multirow{3}{*}{ Boeira (2006) } \\
\hline & $\mathrm{Al}-6,2 \% \mathrm{Cu}$ & $11200(t)^{-0,022}$ & \\
\hline & $\mathrm{Al}-8,1 \% \mathrm{Cu}$ & $10700(t)^{-0,17}$ & \\
\hline \multirow{4}{*}{ Vertical Descendente } & $\mathrm{Al}-3 \% \mathrm{Cu}$ & $2700(t)^{-0,16}$ & \multirow{4}{*}{ Rosa (2004) } \\
\hline & Al-5\%Cu & $2300(t)^{-0,10}$ & \\
\hline & $\mathrm{Al}-8 \% \mathrm{Cu}$ & $5000(t)^{-0,16}$ & \\
\hline & $\mathrm{Al}-15 \% \mathrm{Cu}$ & $17000(t)^{-0,543}$ & \\
\hline
\end{tabular}

No que se refere ao sistema com configuração horizontal, são raros os trabalhos na literatura que abordam o assunto (Silva et al., 2011).

Quando a solidificação é realizada no sentido vertical ascendente, em condições de gradiente de temperatura positivo no líquido, o efeito da convecção é minimizado quando o soluto é direcionado para regiões interdendríticas, promovendo um líquido mais denso que o volume global do metal líquido e uma solidificação mais estável termicamente. No caso da solidificação vertical descendente o fenômeno da conveç̧ão no líquido, devido o soluto rejeitado e o efeito da gravidade, são preponderantes na formação da morfologia da microestrutura frente à interface sólido/liquido, pois, ao contrário da solidificação vertical ascendente, pode ocorrer o fenômeno da convecção termossolutal, isto é, convecção devido ao movimento de soluto à frente desta interface. Por outro lado, na solidificação direcional horizontal, quando o fluxo de calor é extraído através de somente uma das paredes laterais do molde, a convecção em função dos gradientes de composição no líquido, a convecção termossolutal e o efeito da gravidade, estão presentes. Portanto, uma interessante característica adicional do sistema horizontal é o gradiente de concentração de soluto bem como os efeitos de densidade na direção vertical uma 
vez que o líquido enriquecido de soluto sempre decanta ao passo que o solvente tende a emergir devido as forças de flutuabilidade. Além disso, devido os efeitos impostos pela convecção termossolutal, sempre vai ocorrer um gradiente de temperaturas na direção vertical.

Considerando o exposto, o principal objetivo deste trabalho é determinar o coeficiente de transferência de calor na interface metal/molde $\left(h_{i}\right)$ da liga Al-6\%Si solidificada direcionalmente em um sistema horizontal refrigerado a água bem como as correspondentes varáveis térmicas de solidificação velocidade da isoterma liquidus $\left(V_{L}\right)$ e taxa de resfriamento $\left(T_{R}\right)$.

\section{PROCEDIMENTO EXPERIMENTAL}

O sistema de solidificação empregado nos experimentos foi utilizado pela primeira vez por Silva (2007), projetado de tal maneira que o calor do metal líquido fosse extraído somente através de um sistema refrigerado a água, localizado em uma das paredes laterais do molde, promovendo assim a solidificação direcional horizontal. A lingoteira de aço inoxidável utilizada possui $110 \mathrm{~mm}$ de comprimento, $70 \mathrm{~mm}$ de largura, $60 \mathrm{~mm}$ de altura e $3 \mathrm{~mm}$ de espessura. As superfícies laterais internas da mesma foram revestidas com camadas de alumina e a parte superior foi isolada com material refratário para evitar perdas de calor para o meio ambiente. A condição de contato térmico na interface metal/molde foi padronizada com a superfície de extração de calor sendo polida. Alguns experimentos foram inicialmente realizados com o objetivo de aferir-se a direcionalidade horizontal do fluxo de calor por parte do dispositivo de solidificação. O experimento foi realizado com a liga Al-6\%Cu. A Figura 4 mostra de forma esquemática o dispositivo utilizado.

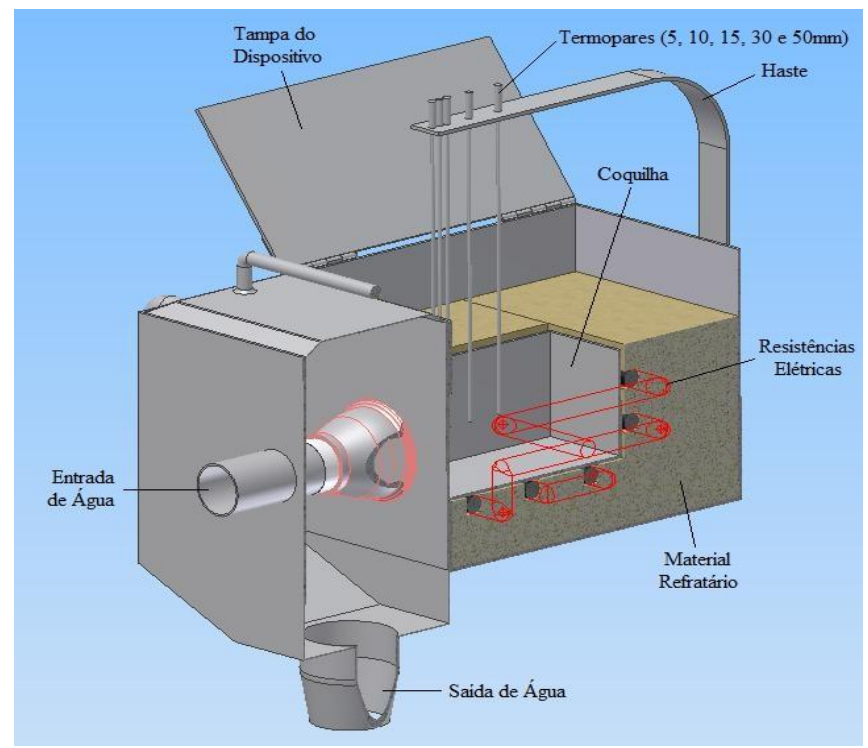

Figura 4 - Representação esquemática do dispositivo de solidificação direcional horizontal utilizado nos experimentos deste trabalho (Silva, 2007).

A liga utilizada foi obtida a partir de lingotes de alumínio e cobre utilizando metais comercialmente puros, cujas composições químicas são apresentadas na Tabela 2. 
Tabela 2 - Análise química dos metais utilizados para preparação da liga Al-6\%Cu.

\begin{tabular}{c|c|c|c|c|c|c|c|c|c|c|c}
\hline \multirow{2}{*}{ Metal } & \multicolumn{10}{c}{ Composição Química (\%) } \\
\cline { 2 - 12 } & $\mathrm{Al}$ & $\mathrm{Fe}$ & $\mathrm{Ni}$ & $\mathrm{Cu}$ & $\mathrm{Si}$ & $\mathrm{Mg}$ & $\mathrm{Pb}$ & $\mathrm{Cr}$ & $\mathrm{Mn}$ & $\mathrm{Zn}$ & $\mathrm{Sn}$ \\
\hline $\mathrm{Al}$ & 99.68 & 0.175 & 0.0148 & 0.0242 & 0.103 & 0.0011 & - & - & - & - & - \\
\hline $\mathrm{Cu}$ & - & - & - & 99.64 & 0.09 & - & 0.002 & 0.27 & - & - & - \\
\hline
\end{tabular}

Para confirmação da concentração de soluto $(6 \% \mathrm{Cu})$, inicialmente a mesma foi caracterizada termicamente, obtendo-se a curva de resfriamento para confirmação dos valores das temperaturas liquidus e solidus, $T_{L}$ e $T_{S}$, respectivamente. Em seguida, os valores observados na curva de resfriamento levantada foram comparados com aqueles representados no diagrama de equilíbrio de fases do sistema Al-Cu. A Figura 5 apresenta o diagrama de equilíbrio de fases parcial do sistema Al-Cu e a curva de resfriamento experimental obtida para a liga $\mathrm{Al}-6 \% \mathrm{Cu}$.

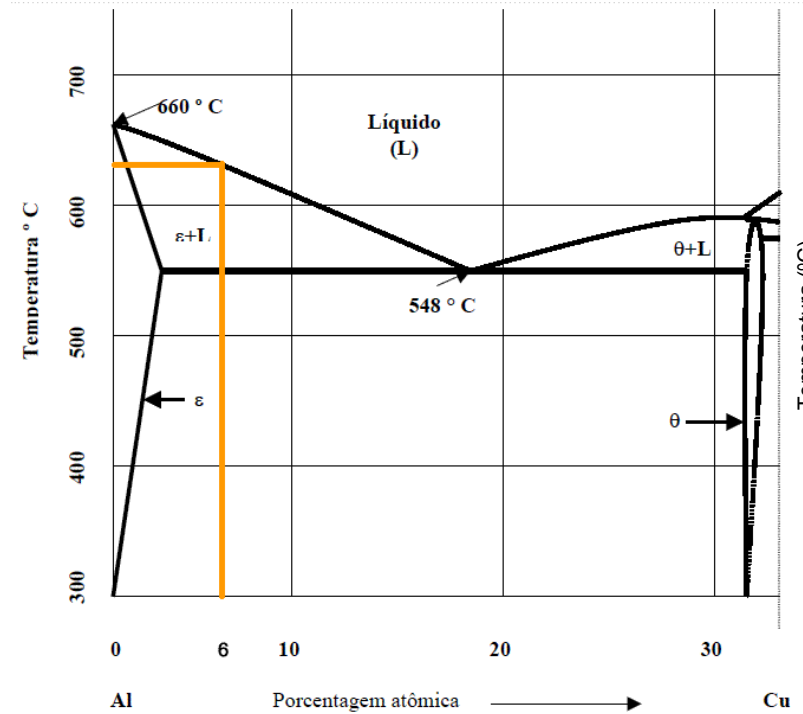

(a)

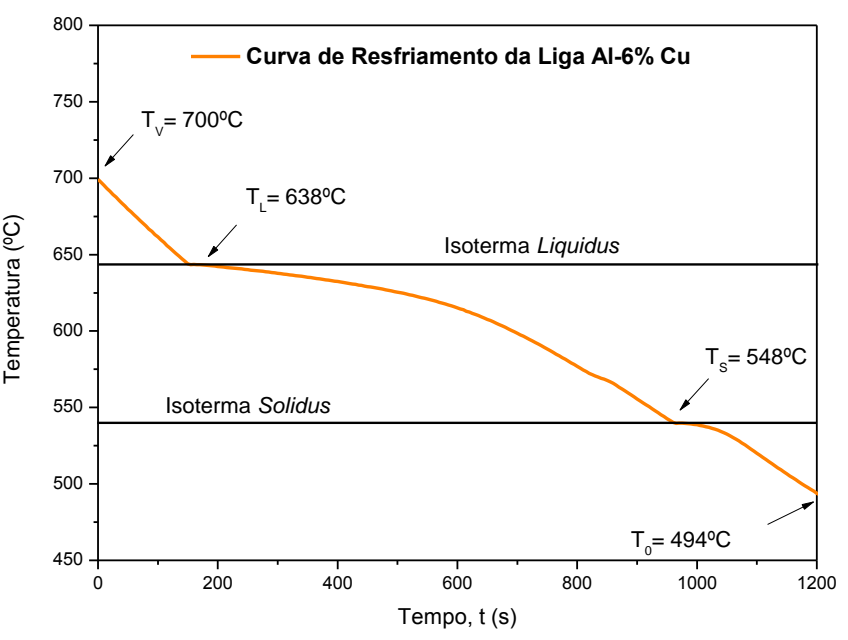

(b)

Figura 5 - (a) Diagrama de equilíbrio parcial do sistema Al-Cu (adaptado de Rocha (2003); (b) curva de resfriamento experimental da liga Al-6\%Cu. $\mathrm{T}_{0}$ é a temperatura final do experimento.

Inicialmente a liga foi fundida em um forno tipo mufla e o metal líquido vazado em uma lingoteira acoplada ao dispositivo de solidificação unidirecional. O sistema de aquecimento do dispositivo foi acionado para garantir a total fusão da liga e a estabilização do líquido, sendo aplicado um superaquecimento de $10 \%$ acima da temperatura liquidus $\left(T_{L}\right)$ da respectiva liga. $A$ partir dessa temperatura, o sistema de aquecimento do dispositivo foi desligado iniciando-se o resfriamento do metal. No momento em que a temperatura do líquido atingiu o patamar para o início do experimento o sistema de refrigeração foi acionado. Essa condição de resfriamento permaneceu até a solidificação do lingote se completar. O monitoramento e os registros térmicos 
foram realizados através de termopares posicionados no interior da lingoteira, conectados a um sistema de aquisição de dados. Neste trabalho, foram utilizados termopares tipo K revestidos por uma bainha de aço inoxidável com diâmetro externo de $1,5 \mathrm{~mm}$. As propriedades termofísicas utilizadas nas simulações para a determinação de $h_{i}$ se encontram apresentadas no trabalho de Boeira e coautores (Boeira et al., 2011).

O sistema de aquisição de dados empregado no experimento acoplado ao dispositivo de solidificação é mostrado de forma esquemática na Figura 6

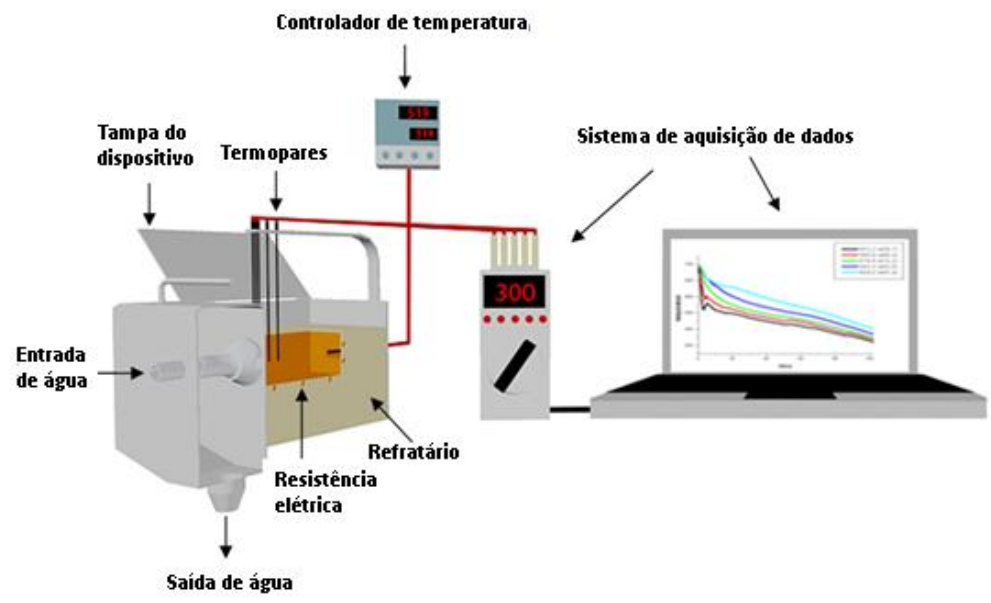

Figura 6 - Representação esquemática do dispositivo de solidificação mostrando sua interface com o sistema de registro de temperaturas.

\section{RESULTADOS E DISCUSSÕES}

A Figura 7 mostra os perfis térmicos de solidificação para os cinco termopares posicionados no metal. O método aplicado para determinação do $h_{i}$ utilizou os arquivos das temperaturas em função do tempo mapeados experimentalmente.

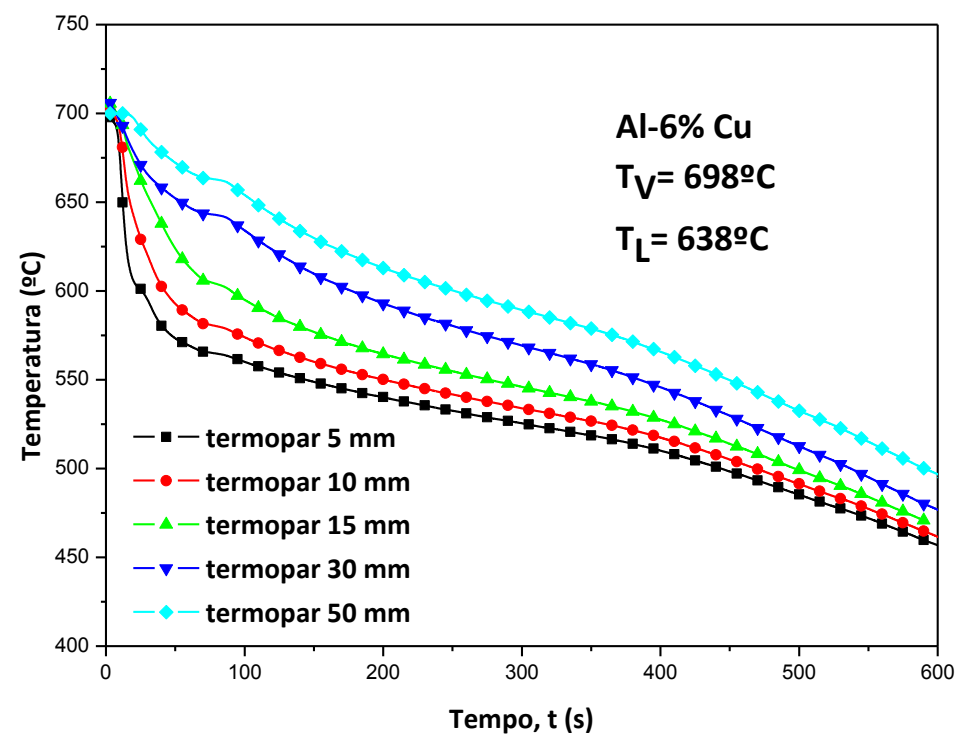

Figura 7 - Perfis de temperatura obtidos experimentalmente para a liga Al-6\%Cu. 
O confronto entre os perfis teóricos e experimentais é mostrado na Figura 8, na qual pode ser verificado que o melhor ajuste entre as curvas ocorreu para o "fit" $h_{i}=3000(t)^{-0,2}$, cujo resultado foi obtido pelo modelo numérico. Observa-se, pela Figura 8, que para a determinação do $h_{i}$, foram utilizados os perfis térmicos referentes aos termopares localizados a 5,10 e $15 \mathrm{~mm}$ da superfície refrigerada onde eventuais perdas de calor através das paredes laterais do molde são mais improváveis o que assegura melhores condições de unidirecionalidade para o fluxo de calor.

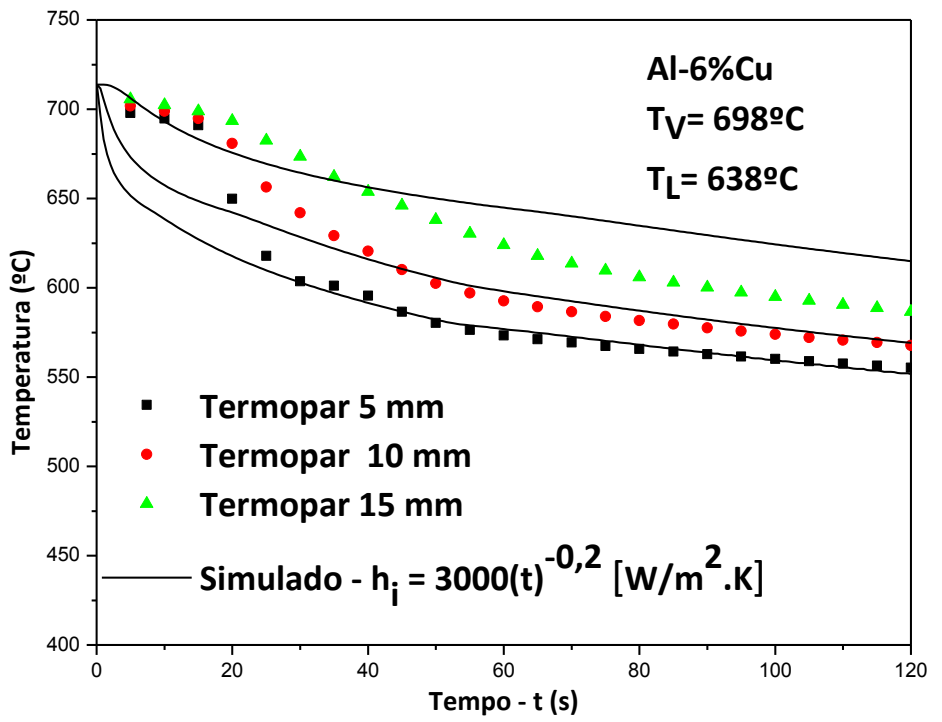

Figura 8 - Curvas teóricas e experimentais de resfriamento correspondentes aos termopares localizados a 5, 10 e $15 \mathrm{~mm}$ a partir da interface metal/molde e o valor de $h_{\mathrm{i}}$.

A Figura 9 apresenta a comparação entre os perfis de $h_{i}$ durante a solidificação horizontal, resultante deste trabalho, e aquele obtido na solidificação vertical ascendente (Boeira, 2006).

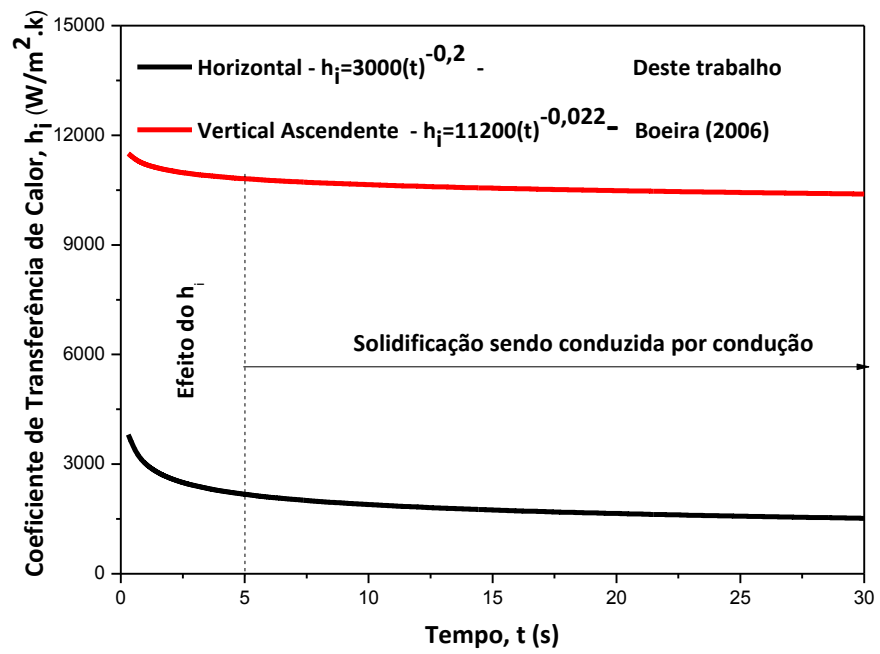

Figura 9 - Coeficientes de transferência de calor em função do tempo para a liga Al-6\%Cu solidificada nas direções horizontal e vertical ascendente.

A partir dos perfis de temperatura da liga estudada, os pontos de intersecção entre a temperatura liquidus e os respectivos perfis permitem determinar, para cada posição de termopar, um par ordenado posição $x$ tempo. Portanto, foram plotados cinco pares ordenados 
da posição em função do tempo os quais aparecem ilustrados no gráfico da Figura 10(a), sendo então obtida uma função potência da posição com o tempo. A derivada desta função em relação ao tempo permitiu o cálculo da velocidade experimental da isoterma liquidus $\left(V_{\mathrm{L}}\right)$, conforme pode ser observado na Figura 10(b). A taxa de resfriamento para cada posição dos termopares foi obtida experimentalmente a partir das interseções da reta correspondente à temperatura liquidus com os perfis térmicos equivalentes a cada posição dos termopares, através do resultado da leitura direta do quociente das temperaturas imediatamente antes e depois da temperatura liquidus e dos tempos correspondentes, isto é, $T_{R}=\Delta T / \Delta t$. Assim, com base nos pontos experimentais foram geradas funções potência da taxa de resfriamento em função da posição cujos resultados encontram-se indicados na Figura 10(c).

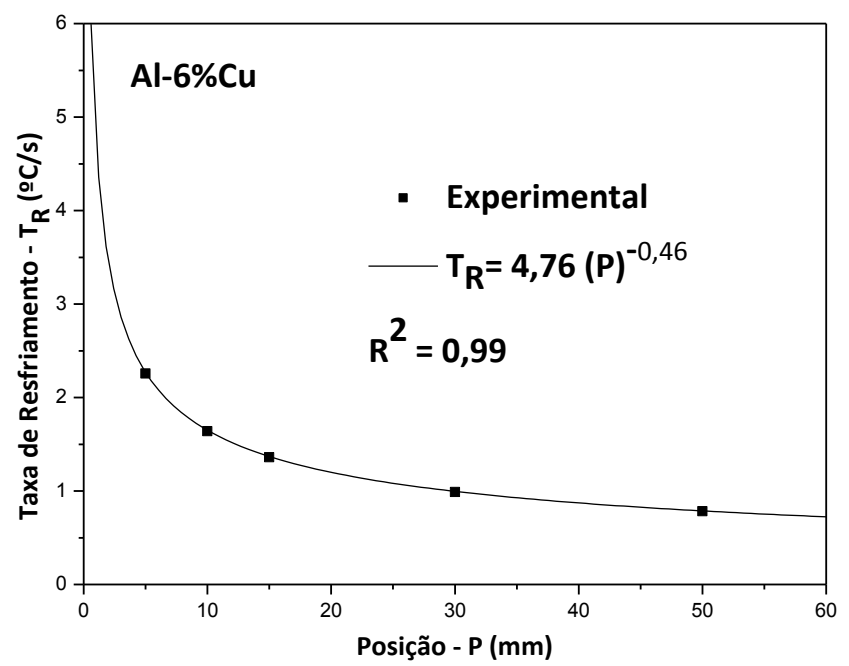

Figura 10 - (a) Posição da isoterma liquidus a partir da interface metal/molde em função do tempo; (b) Velocidades de deslocamento da isoterma liquidus em função da posição da isoterma liquidus; (c) Taxas de resfriamento em função da posição a partir da interface metal/molde.

\section{CONCLUSÕES}

O estudo teórico-experimental foi capaz de prever satisfatoriamente os perfis de $h_{i}$ da liga Al-6\%Cu quando solidificada direcionalmente em um sistema horizontal refrigerado a água sob condições transientes de extração de calor. Os valores de $h_{i}$ podem ser representados por uma equação na forma de potência em função do tempo dada por $h_{i}=3800(t)^{-0,2}$. Os valores experimentais de $V_{L}$ e $T_{R}$ diminuem com o avanço da isoterma liquidus, isto é, quanto mais próximo da superfície de extração de calor maiores são esses parâmetros. Os mesmos podem ser representados por equações na forma de potência em função da posição dadas, respectivamente, por $V_{L}=0,75(P)^{-0,27}$ e $T_{R}=4,76(P)^{-0,46}$. Finalmente, podemos concluir que nos instantes iniciais do processo a extração de calor do sistema metal/molde é conduzida sob o efeito do $h_{i}$ sendo que após os primeiros cinco segundos, devido à formação da camada de sólido, a solidificação passa a ser controlada pelo modo de condução de extração de calor. Entretanto, elaborações de estudos futuros, para outros sistemas de ligas, que permitam avaliar o efeito de $h_{i}$ nas variáveis térmicas de solidificação, são necessários. 


\section{AGRADECIMENTOS}

Os autores agradecem ao Conselho Nacional de Desenvolvimento Científico e Tecnológico (CNPq), à Fundação da Amazônia Paraense (FAP) e ao Instituto Federal de Educação, Ciência e Tecnologia do Pará (IFPA) pelo apoio na concessão de bolsas de Iniciação Científica e de Desenvolvimento Tecnológico para os alunos do IFPA.

\section{REFERÊNCIAS BIBLIOGRÁFICAS}

BOEIRA, A. P. Macrossegregação e Formação de Poros na Solidificação de ligas Al-Cu: Modelagem Numérica e Desenvolvimento Experimental. Campinas, 2006. Tese de Doutorado-Faculdade de Engenharia Mecânica-Universidade Estadual de Campinas, 2006.

FERREIRA, I. L. Análises Numérica, Analítica e Experimental da Macrossegregação Inversa na Solidificação. Campinas, 2004. Tese de Doutorado-Faculdade de Engenharia MecânicaUniversidade Estadual de Campinas, 2004.

GARCIA, A. Solidificação Fundamentos e Aplicações. UNICAMP-SP, 2007.

MEZA, E. S. Modelagem Analítica e Numérica da Solidificação de Ligas Binárias: Análise de Fatores de Influência. Campinas, 2012. Tese de Doutorado-Faculdade de Engenharia MecânicaUniversidade Estadual de Campinas, 2012.

ROCHA, O. L., SIQUEIRA, C. A., GARCIA, A. Heat flow parameters dendrite spacings during unsteady state solidification of $\mathrm{Sn}-\mathrm{Pb}$ and $\mathrm{Al}-\mathrm{Cu}$ alloys. Metallurgical and Materials Transactions A, v.34A, p.995-1006, 2003.

ROSA, D. M. Caracterização da Microestrutura Dendrítica na Solidificação Vertical Descendente de Ligas Al-Cu. Campinas, 2004. Tese de Doutorado-Faculdade de Engenharia MecânicaUniversidade Estadual de Campinas, 2004.

SANTOS, C. A., QUARESMA, J. M. V., GARCIA, A. Determination of Transient Interfacial Heat Transfer Coefficients in Chill Mold Castings. Journal of Alloys and Compounds, v.319, p.174-186, 2001.

SILVA, J. N. S. Projeto, Construção e Aferição de um Dispositivo de Solidificação Unidirecional Horizontal Refrigerado a Água. Dissertação de Mestrado-Instituto de Tecnologia Programa de Pós-Graduação em Engenharia Mecânica-Universidade Federal do Pará, 2007.

SILVA, J.N., MOUTINHO, D.J., MOREIRA, A. L., FERREIRA, I. L., ROCHA, O. L. Determination of Heat Transfer Coefficients at Metal-Mold Interface During Horizontal Unsteady-State Directional Solidification of Sn-Pb Alloys. Materials Chemistry and Physics, v.130, p.179-185, 2011.

SIQUEIRA, C. A. Influência de Parâmetros Térmicos de Solidificação na Transição Colunar/Equiaxial. Campinas, 2002. Tese de Doutorado-Faculdade de Engenharia MecânicaUniversidade Estadual de Campinas, 2002.

SPINELLI, J.E., FERREIRA, I.L., GARCIA, A. Influence of Melt Convection on the Columnar to Equiaxed Transition and Microstructure of Downward Unsteady-State Directionally Solidified Sn$\mathrm{Pb}$ Alloys. Journal of Alloys and Compounds, v.384, p.217-226, 2004. 